\title{
DISTAL RENAL TUBULAR ACIDOSIS AS COMPLICATION OF MIXED CONNECTIVE TISSUE DISORDER WITH AUTOIMMUNE HYPOTHYROIDISM- A CASE REPORT
}

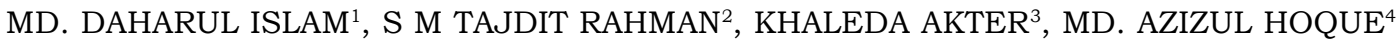

\begin{abstract}
Renal tubular acidosis (RTA) is a constellation of syndromes arising from different derangements of tubular acid transport. Multiple associations have been established with renal tubular acidosis. We report a case of distal renal tubular acidosis which may be associated with undifferentiated connective tissue disease in which patient is also having autoimmune hypothyroidism along with that is not very common.
\end{abstract}

Received: 10 July 2015

Accepted: 3 November 2015

\section{Introduction}

Renal tubular acidosis (RTA) is non-uremic defects of urinary acidification. Renal tubular acidosis is characterized by a normal anion gap hyperchloremic metabolic acidosis; plasma potassium may be normal, low or high-depending on the type of RTA. The metabolic acidosis in distal RTA (dRTA) is characterized by a normal anion gap, urinary $\mathrm{pH}>$ 5.3, and low ammonium excretion. dRTA is usually caused by a proton secretion defect in the intercalated cells. This proton secretion defect explains why dRTA is usually accompanied by hypokalemia, because it indirectly causes renal potassium losses and cellular shifts of potassium. dRTA may be inherited or acquired by drugs, hypercalciuria, or an underlying autoimmune disorder. ${ }^{1}$ It has been reported in various autoimmune disorders including Sjögren's syndrome, primary biliary cirrhosis, autoimmune hepatitis, systemic lupus erythematosus, and rheumatoid arthritis. ${ }^{1}$ How these autoimmune disorders cause dRTA is not well known. dRTA is a relatively common complication of Sjögren's syndrome, and studies have suggested the presence of autoantibodies against acid- base transporters. ${ }^{2,3,4}$ Here, we report a case of dRTA associated with mixed connective tissue disorder with autoimmune thyroiditis who had episodic history of weakness of both upper and lower limb for several times.

\section{Case Report}

A 30 year old female with previous history of neck swelling in the midline presented with progressive muscle weakness of both lower limb for 8-10 hours. She had history of same episodic weakness 3 times for last 1 year. She also had occasional both knee joints pain which is more marked in the morning associated with morning stiffness for 10-15 minutes. Later on, she also developed multiple large joints pain. Her dietary habits were unremarkable and she did not have diarrhea. She used no other medication except potassium supplement for electrolyte imbalance and thyroxin for hypothyroidism. She did not use $\mathrm{H} 2$ receptor antagonists or proton pump inhibitors. Besides muscle weakness (strength 3/5) and knee joint tenderness, physical examination was normal except thyroid gland which was slightly enlarged.

Laboratory tests at admission revealed hypokalemia (serum potassium $2.4 \mathrm{mmol} / \mathrm{L}$ ) and non anion gap metabolic acidosis (serum pH 7.22, bicarbonate $12.4 \mathrm{mmol} / 1$, blood aninon gap $14 \mathrm{mmol} / 1)$. Hypokalemia was due to renal potassium losses (urine potassium $284 \mathrm{mmol} / 24 \mathrm{hr}$ ). The metabolic acidosis was characterized by an inappropriately high urine $\mathrm{pH}$ of 6.7 and a positive urine anion gap (+18mmol/1), suggesting renal tubular acidosis (RTA). She was treated with potassium supplement and her condition developed.

1. Dr. Md. Daharul Islam; Assistant Professor, Department of Medicine, Sir Salimullah Medical College Mitford Hospital, Dhaka

2. Dr. SM Tajdit Rahman; 27, Navana Garden, Kallyanpur, Dhaka.

3. Dr. Khaleda Akter, Assistant Professor, Department of Obstetrics \& Gynecology, Z H Sikder Womens Medical College, Dhaka.

4. Dr. Md. Azizul Hoque, Associate Professor, Department of Endocrinology, Sirajgonj Medical College, Sirajgon

Bangladesh J Medicine 2016; 27 : 37-39 
After the acute phase, additional tests were performed to investigate the type and cause of RTA. An acidification test using fludrocortisone and furosemide, and ammonium chloride was performed during which she failed to reach a urine $\mathrm{pH}<5.3$ (lowest urine pH 5.7). Whereas, renal ammonium excretion was assessed by calculating the urine osmolal gap and found to be very low. All these findings confirmed distal renal tubular acidosis.

Our diagnosis was further supported by the absence of other markers of proximal tubular dysfunction (no hypouricemia, hypophosphatemia, proteinuria or glucosuria), and the absence of paraprotein and urinary light chains. Herditary form of dRTA is also excluded as there is no positive family history and onset is late. Kidney size was normal in sonography.

Further investigations were done to find out systemic autoimmune diseases and cause of hypothyroidism. All the ENA profile showed negative except anti SM/ RNP antibody, ANA (strongly positive) and rheumatoid factor. There was no hypercalciuria. Serum calcium, magnesium, basal cortisol, aldesterone and rennin in plasma were normal. However, thyroid function test shows antibodies against thyroid peroxidase was highly positive otherwise normal. Patient didn't give consent of renal biopsy and a final diagnosis of undifferentiated connective tissue disorder with autoimmune thyroiditis with distal renal tubular acidosis was made.

Later on, she was treated with potassium citrate during discharge. She didn't give any complaints during follow up after 6 months.

\section{Discussion}

The presence of distal type renal tubular acidosis should be suspected in any patient with a normal anion gap, metabolic acidosis, and a urine $\mathrm{pH}$ greater than 5.3 in adults. Many different conditions have been associated with distal type renal tubular acidosis. The major causes of this disorder include primary idiopathic disease, hereditary conditions such as Wilson's disease, disorders of calcium metabolism and nephrol- calcinosis, autoimmune diseases, drugs and toxins, urinary tract obstruction and sickle-cell anaemia ${ }^{5}$. It has been thought that the distal tubular acidosis associated with hyperthyroidism could be a metabolic complication of hyperthyroidism via hypercalcaemia, hypercalciuria, and nephrocalcinosis, which might elicit a giant-cell reaction and develop tubular dysfunction. ${ }^{6}$ In our patient there was no evidence of hypercalcaemia and nephrocalcinosis. However, the concurrence of renal tubular acidosis and autoimmune thyroid disease has been reported before. ${ }^{7}$ Although the mechanism remains unclear, distal tubular acidosis has been well documented in the presence of a variety of autoimmune disorders including thyroid disease, Sjogrens syndrome, systemic lupus erythematosus, etc. The critical question regarding the present report is whether renal tubular acidosis in this patient resulted from an udifferntiated autoimmune disorder or from autoimmune thyoiditis (hypothyroidism).

It is difficult to tell the cause of distal renal tubular acidosis. Treatment with thyroid hormone replacement didn't cure the complication, but lessen some degree. A renal biopsy would have been conclusive regarding the cause of renal tubular acidosis. But there might be overlapping of both undifferentiated connective tissue disorder and autoimmune thyroiditis.

Three mechanisms are possible behind distal renal tubular acidosis: (1) Defect in H+-ATPase pump of the cortical and/or medullary collecting tubules, (2) Reduction in cortical sodium reabsorption, thereby diminishing the degree of luminal negativity and producing a voltage-dependent defect which will lead to a concurrent impairment in potassium secretion and result in hyperkalaemia. A reduction in sodiumpotassium-ATPase activity may be responsible for the reduction in sodium resorption, (3) An increase in membrane permeability, which allows back-diffusion of hydrogen ions or possibly bicarbonate. ${ }^{5,8}$

The mechanisms underlying hypokalemia in distal renal tubular acidosis have not been fully elucidated. The hypokalemia is particularly prevalent in the acquired forms. Hypokalemia probably results from increased potassium excretion due to renal tubular leakage, decreased proximal tubular reabsorption in the face of acidosis and hypocapnia, and aldosterone stimulation. ${ }^{9}$ It is important to differentiate from other causes of hypokalemia like familial periodic paralysis (FPP), thyrotoxicosis, hyperaldosteronism and gastrointestinal loss; because the treatment is different.

The concurrence of renal tubular acidosis and autoimmune thyroid disease has been reported before. Although the mechanism remains unclear, distal renal tubular acidosis has been well documented in the presence of a variety of autoimmune disorders including thyroid disease, Sjogrens syndrome and systemic lupus erythematosus. Symptoms of renal tubular acidosis usually resolve rapidly with substitution of thyroid hormone. ${ }^{6}$ However, autoimmune primary hypothyroidism in our patient was evidenced by the high titer of antithyroid peroxidase and did not resolve 
after thyroid hormone replacement, which implies of its non-association. But our patient has undifferentiated connective tissue disorder which may be the underlying cause.

There was a negative family history in our case; however a negative family history does not exclude autosomal dominant (AD) inheritance. Growth retardation is common in $\mathrm{AD}$ inheritance. Although we could not perform genetic analysis; the possibility of $\mathrm{AD}$ dominant inheritance in this case cannot be excluded.

\section{Conclusion}

We report an unusual case of dRTA, which was clearly of autoimmune origin, but could not be linked to any known systemic autoimmune disorder known to be associated with dRTA. But this case has autoimmune thyroiditis and undifferentiated connective tissue disease, which may be a cause of distal renal tubular acidosis. So we may find renal tubular acidosis as a complication of undifferentiated connective tissue disorder or autoimmune thyroiditis.

\section{References}

1. Rodriguez Soriano J. Renal tubular acidosis: the clinical entity. J Am Soc Nephrol. 2002;13(8): 2160-70.

2. Cohen EP, Bastani B, Cohen MR, Kolner S, Hemken $\mathrm{P}$, Gluck SL. Absence of $\mathrm{H}(+)$-ATPase in cortical collecting tubules of a patient with Sjogren's syndrome and distal renal tubular acidosis. J Am Soc Nephrol. 1992;3(2):264-71.

3. Walsh S, Turner CM, Toye A, Wagner C, Jaeger P, Laing $\mathrm{C}$, et al. Immunohistochemical comparison of a case of inherited distal renal tubular acidosis (with a unique $\mathrm{AE} 1$ mutation) with an acquired case secondary to autoimmune disease. Nephrol Dial Transplant. 2007;22(3):807-12.

4. Han JS, Kim GH, Kim J, Jeon US, Joo KW, Na KY, et al. Secretory-defect distal renal tubular acidosis is associated with transporter defect in $\mathrm{H}(+)$-ATPase and anion exchanger-1. J Am Soc Nephrol. 2002;13(6):1425-32.

5. Rose BD. Clinical Physiology of Acid-Base and Electrolyte Disorders. McGraw-Hill, New York, 1994; 572-586.

6. Mason AM, Golding PL. Renal tubular acidosis and autoimmune thyroid disease. Lancet 1970; 2: 11041106.

7. Jaeger P, Partmann L, Wauters JP et al. Distal renal tubular acidosis and lymphocytic thyroiditis with spontaneously resolving hyperthyroidism. Am J Nephrol 1985; 5: 116-120

8. Kurtzman NA. Disorders of distal acidification. Kidney Int 1990;38:720-727.

9. Blake-Palmer KG, Karet FE. Cellular physiology of the renal H+ATPase. Curr Opin Nephrol Hypertens 2009. Sep;18(5):433-438. 